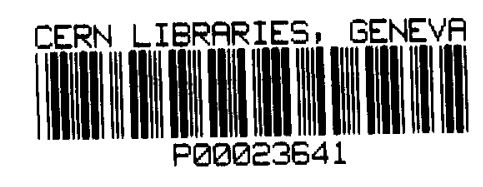

VAND-TH-94-10

April 1994

\section{The Neutralino Sector in Strongly Interacting} Supersymmetric Models

W.T.A. ter Veldhuis

Department of Physics and Astronomy

Vanderbilt University

Nashville, TN 37235

\section{Abstract}

The neutralino sector is analyzed for a supersymmetric non-linear sigma model. This model describes the low energy physics of strongly interacting theories in which supersymmetry is softly broken at scales below the electroweak symmetry breaking scale. The measured width of the $\mathrm{Z}$ boson constrains the allowed range of parameters. In case the lightest neutralino is stable, limits on additional contributions to the invisible width of the Z boson and on the relic neutralino density further restrict parameter space. As a consequence, the lightest neutralino in the considered class of theories is required to have a mass above $15 \mathrm{GeV}$, but below $M_{Z}$

\section{VAND - TH $94-10$ \\ fur 9425}

\section{Introduction}

Supersymmetry (SUSY) is indispensable in attempts to unify gravity with the strong, weak and electromagnetic forces[1]. Unfortunately, local supersymmetric quantum field theories are not renormalizable; therefore, they defy a consistent interpretation. Nevertheless, it is plausible that a global supersymmetric quantum field theory describes physics at a lower energy scale. If this global supersymmetry is broken only softly, the improved ultraviolet behavior inherent to supersymmetric models is maintained. Eventually, below some energy scale, the successful Standard Model (SM) emerges as a low energy effective theory.

The most popular and minimal linear model to realize such a scenario is the Minimal Supersymmetric Standard Model (MSSM)[2]. The successful unification of the $U(1), S U(2)$ and $S U(3)$ gauge coupling constants, and of the tau lepton and bottom quark Yukawa couplings support the notion of the MSSM embedded in a Grand Unified Theory (GUT)[3]. The supersymmetry breaking scale required for this unification to occur is of the order of $1 \mathrm{TeV}$, indicating intriguing new physics just outside the currently accessible energy range of particle accelerators. Moreover, the ensuing unification scale is of the order of $10^{16} \mathrm{GeV}$, sufficiently large to suppress the proton decay rate to an acceptable level, yet smaller than the Planck scale.

However, the MSSM embedded in a GUT necessitates the existence of a so called desert, the vast energy region between the unification scale and the supersymmetry breaking scale, in which presumably no new physics manifests itself. Alternatively, in the absence of the unification requirement, the minimal supersymmetric $S U(2) \times U(1)$ invariant theory is a gauged nonlinear sigma model $[4,5,6]$. The simplest non-linear action can be cast into a 
form identical to the action of the MSSM, with the additional constraint

$$
H_{T} \in H_{B} \equiv \frac{1}{2} v_{T} v_{B}
$$

This nonlinear model (nISSM) features the same number of parameters as the MSSM, but contains less particles. Specifically, the constraint effectively eliminates the pseudo scalar, one Higgs boson and one of the Higgsinos. In addition, it prevents a $\mu H_{T} \epsilon H_{B}$ term from appearing in the action. The non-renormalizable character forces the interpretation of an effective theory with a momentum cut-off $\Lambda \sim 4 \pi v$. In principle, gauge invariant terms of any mass dimension and with arbitrary coefficients can be added to this action. However, other terms of the lowest chiral dimension in general directly violate $\rho=1[5]$; therefore, the unspecified underlying theory should provide a mechanism for their suppression. Terms of higher chiral dimension on the other hand are assumed to be down by appropriate factors of $1 / \Lambda$. It is thus possible to analyze the low energy dynamics of this model without referring to the particular dynamics that gives rise to it.

In contrast with the SM, which yields the non-linear Higgs-less theory in the formal heavy Higgs limit, the minimal non-linear supersymmetric model is not a limiting form of the MSSM. However, it can be derived from the $(M+1) S S M$, the MSSM with an additional gauge singlet, as this model has independent dimensionless coupling constants in the Higgs sector. The enhancement of longitudinal vector-boson scattering[5] is a striking consequence of the non-linear realization of the symmetry. Both the mass of the neutral Higgs boson, $M_{h}^{2}=M_{Z}^{2}+2 a \sin ^{2} 2 \beta$, and the mass of the charged Higgs boson, $M_{h^{ \pm}}^{2}=M_{W}^{2}+2 a$, depend on the soft SUSY breaking parameter $a$. The strength of this and all other soft SUSY breaking parameters is characterized by $M_{Z}$. Henceforth, supersymmetry is broken at scales much below the elec- troweak breaking scale, and at the same time the masses of the superpartners may differ substantially from their observed counterparts. It is clear that the mass of the neutral Higgs boson is not required to be below $M_{Z}$ at tree level, contrary to the situation in the MSSM.

In the present paper we analyze the neutralino sector of the model in more detail. In particular, we determine the section of parameter space that is ruled out by experimental results and observations. The measurement of the width of the $\mathrm{Z}$ boson at LEP puts limits on the decay rates of the $\mathrm{Z}$ boson into charginos (i) and neutralinos (ii). Furthermore, the R-parity invariance of the non-linear model implies that the lightest supersymmetric particle (LSP) is stable. With the added assumption that the lightest neutralino is the LSP, the parameter space is further restricted by the requirements that this neutralino is lighter than the lightest chargino (iii), the invisible width of the $\mathrm{Z}$ boson does not exceed its LEP bound (iv), and the relic neutralino density does not exceed bounds derived from cosmological and astrophysical considerations ( $v$ ). In the MSSM, relations between soft SUSY breaking gaugino masses do exist when embedded in a GUT. Obviously, as the non-linear model is an effective theory providing a valid description of the physics only in a limited energy range, these relations do not apply here. Thus, the unknown parameters specifying the mass spectrum of the charginos and neutralinos and their interactions are the soft SUSY breaking gaugino masses $\tilde{m}_{Y}$ and $\tilde{m}_{W}$, and $\tan \beta$ 


\section{The neutralino sector}

In the non-linear model the Higgs component fields have to satisfy the constraint[5]

$$
A_{T} \epsilon \psi_{B}+\psi_{T} \epsilon A_{B}=0
$$

As a consequence one Higgsino is eliminated, and the fermionic components of the Higgs superfields may be written to leading order as

$$
\psi_{B}=i\left(\begin{array}{c}
\psi_{+} \\
-\sin \beta \psi_{3}
\end{array}\right) \quad \psi_{T}=i\left(\begin{array}{c}
\cos \beta \psi_{3} \\
\psi_{-}
\end{array}\right) .
$$

In general, the remaining Higgsino mixes with the superpartners of the photon and the $\mathrm{Z}$ boson, and the resulting neutralino mass matrix in the basis $\left(\lambda_{Z}, \psi_{3}, \lambda_{\gamma}\right)$ is given by

$$
\tilde{M}=\left(\begin{array}{ccc}
\tilde{m}_{z} & M_{Z} & \tilde{m}_{\gamma Z} \\
M_{Z} & 0 & 0 \\
\tilde{m}_{\gamma Z} & 0 & \tilde{m}_{\gamma}
\end{array}\right),
$$

where the soft SUSY breaking parameters satisfy

$$
\begin{array}{r}
\tilde{m}_{\gamma}=\left(\tilde{m}_{W} \sin ^{2} \theta_{W}+\tilde{m}_{Y} \cos ^{2} \theta_{W}\right) \\
\tilde{m}_{Z}=\left(\tilde{m}_{W} \cos ^{2} \theta_{W}+\tilde{m}_{Y} \sin ^{2} \theta_{W}\right) \\
\tilde{m}_{\gamma Z}=\frac{1}{2}\left(\tilde{m}_{W}-\tilde{m}_{Y}\right) \sin 2 \theta_{W} .
\end{array}
$$

As opposed to the situation in the MSSM, the neutralino mass matrix is independent of $\tan \beta$. The trace and the determinant of the squared mass matrix yield the neutralino mass relations

$$
\begin{aligned}
\tilde{M}_{1}^{2}+\tilde{M}_{2}^{2}+\tilde{M}_{3}^{2}-2 M_{Z}^{2} & =\tilde{m}_{Z}^{2}+2 \tilde{m}_{\gamma Z}^{2}+\tilde{m}_{\gamma}^{2} \\
\tilde{M}_{1} \tilde{M}_{2} \tilde{M}_{3} & =M_{Z}^{2} \tilde{m}_{\gamma} .
\end{aligned}
$$

In addition, at least one of the neutralinos has a mass above, and at least one has a mass below $M_{Z}$, as can be verified by analyzing the eigenvalues of the two by two submatrices of the squared mass matrix. It is convenient to write the neutralino mass eigenstates as linear combinations of the zino, Higgsino and the photino

$$
\chi_{i}=N_{i Z} \lambda_{z}+N_{i 3} \psi_{3}+N_{i \gamma} \lambda_{\gamma}
$$

where $i=1,2,3$ and $\tilde{M}_{1} \leq \tilde{M}_{2} \leq \tilde{M}_{3}$. The neutralino spectrum depends on only two parameters: $\tilde{m}_{W}$ and $\tilde{m}_{Y}$. We therefore show the mass of the lightest neutralino $\chi_{1}$ and its composition as contours in the $\left(\tilde{m}_{W}, \tilde{m}_{Y}\right)$ plane for the physically distinct cases that $\tilde{m}_{W}$ and $\tilde{m}_{Y}$ have identical, figure 1 , and opposite sign, figure 2 , respectively. The discontinuity in figure 1 indicates a line in parameter space along which the masses of the two lightest neutralinos $\chi_{1}$ and $\chi_{2}$ are degenerate. The composition of $\chi_{1}$ determines its interaction with other particles; for instance, neutralinos only couple to the $\mathrm{Z}$ boson through their Higgsino component.

\section{Z boson decay}

The neutralino and chargino sectors of SUSY models are subject to the constraints from LEP experiments $[7,8]$. In particular, the measurements of the total and visible width of the $\mathrm{Z}$ boson result in bounds on neutralino masses. Since its neutralino spectrum and interactions differ substantially from the MSSM, it is interesting to determine how the electroweak precision data constrains the nISSM.

The non-observation at LEP of the process $Z \rightarrow \chi^{+} \chi^{-}$puts the stringent bound $\tilde{M}_{ \pm}>\frac{1}{2} M_{Z}$ on the mass of charginos. This bound is particularly 
restrictive in the nISSM, because the mass of the lightest chargino is required to be below $M_{W}$ at tree level. Moreover, the processes $Z \rightarrow \chi_{a} \chi_{b}$ yield an additional contribution to the total width of the $\mathrm{Z}$ boson, if kinematically allowed. However, such additional contributions have to be in agreement with the Z-line shape measurements. In addition, if the lightest neutralino $\chi_{1}$ is stable, then the process $Z \rightarrow \chi_{1} \chi_{1}$ contributes to the invisible width of the $\mathrm{Z}$ boson. It is well known that three species of neutrinos very accurately account for the difference between the total width and the visible width; additional contributions to the invisible width are required to be small.

In general, the partial width of a $\mathrm{Z}$ boson decaying into two neutralinos in the nISSM is given by

$$
\begin{aligned}
\Gamma\left(Z \rightarrow \chi_{a} \chi_{b}\right) & =\left(\begin{array}{c}
1, a=b \\
\frac{1}{2}, a \neq b
\end{array}\right)\left(g_{1}^{2}+g_{2}^{2}\right) \frac{M_{Z}}{48 \pi}\left(N_{a 3} N_{b 3}\right)^{2} \cos ^{2} 2 \beta \\
& \times\left[1-\left(\frac{\tilde{M}_{a}+\tilde{M}_{b}}{M_{Z}}\right)^{2}+\frac{1}{2}\left(\frac{\tilde{M}_{a}-\tilde{M}_{b}}{M_{Z}}\right)^{2}-\frac{1}{2}\left(\frac{\tilde{M}_{a}^{2}+\tilde{M}_{b}^{2}}{M_{Z}^{2}}\right)^{2}\right] \\
& \times\left[1-2\left(\frac{\tilde{M}_{a}^{2}+\tilde{M}_{b}^{2}}{M_{Z}^{2}}\right)+\left(\frac{\tilde{M}_{a}^{2}-\tilde{M}_{b}^{2}}{M_{Z}^{2}}\right)^{2}\right]^{1 / 2}
\end{aligned}
$$

if kinematically allowed. This result will be exploited in section 5 to construct the allowed parameter space in the neutralino sector of the nISSM.

\section{Neutralino annihilation}

Mounting evidence from large scale dynamics, notably the observed flatness of rotation curves in spiral galaxies, indicates that visible matter makes up only a fraction of the total energy density in the universe[9]. The question what shape the non-luminous matter takes is an issue of ongoing speculation[10].
In SUSY models the lightest neutralino constitutes a prime dark matter (DM) candidate if it is the LSP $[11,12]$; it interacts only weakly, and it is stable. The relic neutralino density has been studied in the MSSM, both within[13,14] and outside[15] the context of a GUT, and in extensions of the minimal model, such as the $(M+1) \operatorname{SSM}[16,17]$. Over large sections of parameter space neutralinos give a significant contribution to the dark matter density, while at the same time being in agreement with limits on the total matter density as derived from estimates of the age of the universe.

In an expanding and cooling universe, each SUSY partner disappears in turn when the temperature falls below its mass. However, a stable neutralino does not decay; the number of stable neutralinos can only decrease through pair annihilation, a weak process that is not very efficient. Indeed, when the temperature falls below the so called "freeze out temperature" the annihilation rate becomes so low compared to the expansion rate of the universe that the neutralinos cease to maintain chemical equilibrium. Below this temperature their number stays approximately constant. The relic neutralino density may therefore be much higher than naively expected.

Below the freeze out temperature, the neutralinos remain in kinetic equilibrium through interactions with other particles such as electrons. They are now non-relativistic, since the freeze out temperature is typically only a fraction of the neutralino mass.

The annihilation of neutralinos through $\mathrm{Z}$ boson and sfermion exchange is described by the effective lagrangian

$$
\mathcal{L}=\sum_{f} \bar{\chi} \gamma^{\mu} \gamma_{5} \chi \bar{f} \gamma^{\mu}\left(A_{f} P_{L}+B_{f} P_{R}\right) f
$$

where the sum is over all kinematically accessible fermions. As long as the exchanged momenta are small compared to the mass of the $\mathrm{Z}$ boson and the 
sfermion masses, the coefficients $A_{f}$ and $B_{f}$ in the nISSM read

$$
\begin{aligned}
A_{f}^{Z} & =N_{a 3}^{2} \cos 2 \beta \frac{\sqrt{g_{1}^{2}+g_{2}^{2}}}{4 M_{Z}^{2}}\left(Y_{f_{L}} g_{1} \sin \theta_{W}+T_{f_{L}}^{3} g_{2} \cos \theta_{W}\right), \\
B_{f}^{Z} & =N_{a 3}^{2} \cos 2 \beta \frac{\sqrt{g_{1}^{2}+g_{2}^{2}}}{4 M_{Z}^{2}} Y_{f_{R}} g_{1} \sin \theta_{W}, \\
A_{f}^{j} & =\frac{\left(T_{f_{L}}^{3} N_{a W} g_{2}-Y_{f_{L}} N_{a Y} g_{1}\right)^{2}}{2 m_{\bar{f}_{L}}^{2}}-N_{a 3}^{2} \frac{m_{f}^{2}}{m_{\tilde{f}_{L}}^{2}} \frac{g_{1}^{2}+g_{2}^{2}}{8 M_{Z}^{2}}, \\
B_{f}^{j} & =-\frac{\left(Y_{f_{R}} N_{a Y} g_{1}\right)^{2}}{2 m_{\tilde{f}_{R}}^{2}}+N_{a 3}^{2} \frac{m_{f}^{2}}{m_{\tilde{f}_{L}}^{2}} \frac{g_{1}^{2}+g_{2}^{2}}{8 M_{Z}^{2}},
\end{aligned}
$$

with

$$
\begin{aligned}
N_{a W} & =\left(N_{a \gamma} \sin \theta_{W}+N_{a Z} \cos \theta_{W}\right) \\
N_{a Y} & =\left(N_{a \gamma} \cos \theta_{W}-N_{a Z} \sin \theta_{W}\right)
\end{aligned}
$$

The relic density is governed by a Boltzman equation $[18,19]$ which incorporates both the neutralino annihilation rate and the expansion rate of the universe. In the next section we will integrate this equation and thus calculate the current neutralino density.

\section{Results}

In figures 3 and 4 we show the consequences of various constraints in the ( $\tilde{m}_{W}, \tilde{m}_{Y}$ ) plane for several values of $\beta$. Without loss of generality, we choose to show our results for $\beta \leq \pi / 4$; the neutralino mass matrix is independent of $\beta$, while the partial width $\Gamma\left(Z \rightarrow \chi_{a} \chi_{b}\right)$, the neutralino annihilation cross section and the chargino masses depend on $\beta$ only through $\cos 2 \beta$. We proceed to discuss the constraints: (i) The requirement $\tilde{M}_{ \pm}>1 / 2 M_{Z}$ severely reduces the allowed parameter space. In fact, only values of $\beta \in[\pi / 8,3 \pi / 8]$ are allowed. Dashed lines in figures 3 and 4 indicate contours where $\tilde{M}_{ \pm}=1 / 2 M_{Z}$.

(ii) Additional contributions to the width of the $\mathrm{Z}$ boson are limited to $\Gamma_{Z}^{\prime}<0.064 \mathrm{GeV}$ [7]. The decay $Z \rightarrow \chi_{2} \chi_{2}$ is kinematically forbidden in the entire considered parameter space, and the partial widths $\Gamma(Z \rightarrow$ $\left.\chi_{1} \chi_{1}\right)$ and $\Gamma\left(Z \rightarrow \chi_{2} \chi_{1}\right)$ nowhere exceed the experimental bound

(iii) Additional contributions to the invisible width of the $\mathrm{Z}$ boson are limited to $\Gamma_{Z}^{i n v^{\prime}}<13.6 \mathrm{MeV}$ [7]. The partial width $\Gamma\left(Z^{\prime} \rightarrow \chi_{1} \chi_{1}\right)$, which contributes to the invisible width if $\chi_{1}$ is stable, is only significant in regions of parameter space where the lightest neutralino has a large Higgsino component. In addition, this decay mode is suppressed if $\beta$ has a value close to $\pi / 4$. In case $\tilde{m}_{Y}$ and $\tilde{m}_{W}$ have the same sign, the decay is kinematically forbidden in regions of parameter space where the neutralino is mostly a Higgsino. Dot-dashed lines in figures 3 and 4 denote contours along which $\Gamma\left(Z \rightarrow \chi_{1} \chi_{1}\right)=13.6 \mathrm{MeV}$.

(iv) For consistency, in case the lightest neutralino is the LSP, it has to be lighter than the lightest chargino. Solid lines in figures 3 and 4 show contours along which $\tilde{M}_{ \pm}=\tilde{M}_{1}$.

(v) Typically the matter density in the universe is expected to be in the range $0.25<\Omega h_{0}^{2}<0.5$, where $\Omega=\rho / \rho_{c}$ and $h_{0}$ is the Hubble constant $H_{0}$ in units $100 \mathrm{~km} / \mathrm{s} / \mathrm{Mpc}$. This range reflects both a theoretical prejudice in favour of $\Omega=1$ to accommodate inflation and the experimental uncertainty in $h_{0}$. The critical matter density for a flat 
universe is $\rho_{c} \equiv 3 H_{O}^{2} / 8 \pi G=1.9 \times 10^{-29}\left(h_{0}^{2}\right) \mathrm{g} / \mathrm{cm}^{3}$. We use the formalism developed in ref. $[13,19]$ to integrate the Boltzman equation and to calculate the relic neutralino density. For simplicity we assume a common sfermion mass $m_{j}=150 \mathrm{GeV}$. For smaller sfermion masses, the annihilation rate increases. Accordingly, the relic density is lower and in general lighter neutralinos are allowed.

Dotted lines in figures 3 and 4 signify contours along which the relic density saturates the conservative bound $\rho_{\mathrm{X}}=\rho_{c}$ for $h_{0}=0.5$. Light neutralinos tend to yield a relatively high relic density. They freeze out at a low temperature; therefore, they are less diluted by the expansion of the universe. A neutralino with a large Higgsino component results in a large relic density when $\beta \approx \pi / 4$. A pure Higgsino annihilates only through $\mathrm{Z}$ boson exchange, which is suppressed for this value of $\beta$.

The effective lagrangian (4.1) is only valid for $\tilde{M}_{1}^{0} \ll<M_{Z}$. This approximation is consistent, as only light neutralinos are excluded by the relic density constraint. The presence of other particles with masses close to the LSP can substantially enhance the annihilation rate[20]. However, it is not likely that coannihilation will change the results, since both the next to lightest neutralino and the lightest chargino are much heavier in the excluded region.

In conclusion, the requirement that the lightest chargino has to be heavier than half the $\mathrm{Z}$ boson mass greatly reduces the parameter space of the nISSM. Bounds on the visible and invisible width of the $\mathrm{Z}$ boson do not further restrict parameter space, and will not do so unless these bounds are tightened by at least an order of magnitude. Parameter space is further reduced with the additional assumption that the lightest neutralino is the
LSP. In particular, a light neutralino $\tilde{M}_{1}<15 \mathrm{GeV}$ is excluded because the resulting relic density is too high. Note however that a very light neutralino with $M_{x} \sim 100 \mathrm{eV}$ is not excluded.

\section{Acknowledgements}

The author is grateful to Thomas E. Clark, Marco A. Diaz and Thomas E. Weiler for useful discussions. This work was supported by the U.S. Department of Energy under grant No. DE-FG05-8SER40226. 


\section{References}

[1] D.Z. Freedman, P. van Niewenhuizen, S. Ferrara, Phys. Rev. D16 (1976) 3214 ;

S. Deser, B. Zumino, Phys. Lett. 62B (1976) 335;

P. van Niewenhuizen, Phys. Rep. 68C (1981) 189.

[2] H. Nilles, Phys. Rep. 110 (1984) 1;

H.E. Haber and G.L. Kane, Phys. Rep. 117 (1985) 75;

R. Barbieri, Riv. Nuovo Cim. 11 (1988)1.

[3] W. de Boer, Karlsruhe Preprint IEKP-KA/94-01. Presented at the Herbstschule Maria Laach, Maria Laach (1992), and at the HeisenbergLandau Summerschool, Dubna (1992). To appear in Progress in Particle and Nuclear Physics.

[4] S. Ferrara, A. Masiero and M. Poratti, Phys. Lett. B301 (1993) 358; S. Ferrara and A. Masiero, CERN Preprint CERN TH-6846-93, to appear in the Proceedings of SUSY 93, World Scientific, ed. by P. Nath; S. Ferrara, A. Masiero, M. Porrati and R. Stora, CERN Preprint CERN TH-6845-93.

[5] T.E. Clark and W.T.A. ter Veldhuis, Purdue Preprint PURD-TH-9314.

[6] K.J. Barnes, D.A. Ross and R.D. Simmons, Southampton Preprint SHEP 93/84-12.

[7] K. Hidaka, Phys. Rev. D44 (1991) 927.
[8] R. Barbieri, G. Gamberini, G.F. Guidice and G. Ridolfi, Nucl. Phys. B296 (1988) 75 .

[9] E.W. Kolb and M.S. Turner, The Early Universe (Addison Wesley, Redwood City, CA)

[10] M.S. Turner, FERMILAB-CONF-92-382-A. Presented at NAS Special Colloq. on Physical Cosmology, Irvine, CA, Mar 1992. To appear in Proc. of National Academy of Sciences.

[11] L. Roszkowski, UM-TH-93-06. Presented at 32nd Cracow School on Theoretical Physics, Zakopane, Poland, Jun 2-12, 1992, and at 23rd Workshop Properties of SUSY Particles, Erice, Italy, Sep 28 - Oct 4, 1992, and at 1993 Aspen Winter Conf. on High-Energy Physics, Aspen, CO, Jan 11-16, 1993. To appear in the Proc. of the Erice Workshop.

[12] M. Drees, MAD-PH-818. Presented at International Conference on Non-Accelerator Particle Physics - ICNAPP, Bangalore, India, 2-9 Jan 1994.

[13] J. Ellis, J.S. Hagelin, D.V. Nanopoulos, K.A. Olive and M. Srednicki, Nucl. Phys. B238 (1984) 453.

[14] M. Drees and M.M. Nojiri, Phys. Rev. D47 (1993) 376.

[15] K. Griest and L. Roszkowski, Phys. Rev. D46 (1992) 3309.

[16] R. Flores, K.A. Olive and D. Thomas, Phys. Lett. B245 (1990) 509.

[17] K.A. Olive and D. Thomas, Nucl. Phys. B355 (1991) 192.

[18] M. Srednicki, R. Watkins and K.A. Olive, Nucl. Phys. B310 (1988) 693. 
[19] B.W. Lee and S. Weinberg, Phys. Rev. Lett. 39 (1977) 165.

[20] S. Mizuta and M. Yamaguchi, Phys. Lett. B298 (1993) 120.

\section{FIGURE CAPTIONS}

Fig. 1. Mass and composition contours of the lightest neutralino in the ( $\tilde{m}_{W}, \tilde{m}_{Y}$ ) plane for the case $\tilde{m}_{W}$ and $\tilde{m}_{Y}$ have like sign. (a) Contours of constant $\tilde{M}_{1} / M_{Z}$. (b) Contours of constant $N_{1 Z}^{2}$. (c) Contours of constant $N_{13}^{2}$. (d) Contours of constant $N_{1 \gamma}^{2}$.

Fig. 2. Mass and composition contours of the lightest neutralino in the ( $\tilde{m}_{W}, \tilde{m}_{Y}$ ) plane for the case $\tilde{m}_{W}$ and $\tilde{m}_{Y}$ have opposite sign. (a) Contours of constant $\tilde{M}_{1} / M_{Z}$. (b) Contours of constant $N_{1 Z}^{2}$. (c) Contours of constant $N_{13}^{2}$. (d) Contours of constant $N_{1 \gamma}^{2}$.

Fig. 3. Allowed (hatched) regions in the $\left(\tilde{m}_{W}, \tilde{m}_{Y}\right)$ plane for the case $\tilde{m}_{W}$ and $\tilde{m}_{Y}$ have like sign for (a) $\beta=\pi / 4$, (b) $\beta=\pi / 5$, (c) $\beta=\pi / 6$ and (d) $\beta=\pi / 7$. Dashed lines indicate $\tilde{M}_{ \pm}=1 / 2 M_{Z}$, dotted lines denote $\rho_{\chi}=\rho_{c}$, dot-dashed lines signify $\Gamma\left(Z \rightarrow \chi_{1}^{0} \chi_{1}^{0}\right)=\Gamma_{Z}^{i n u^{\prime}}$, and along the solid lines $\tilde{M}_{1}=\tilde{M}_{ \pm}$. Arrows point towards the region allowed by each individual constraint.

Fig. 4. Allowed (hatched) regions in the $\left(\tilde{m}_{W}, \tilde{m}_{Y}\right)$ plane for the case $\tilde{m}_{W}$ and $\tilde{m}_{Y}$ have opposite sign for (a) $\beta=\pi / 4$, (b) $\beta=\pi / 5$, (c) $\beta=\pi / 6$ and (d) $\beta=\pi / 7$. Dashed lines indicate $\tilde{M}_{ \pm}=1 / 2 M_{Z}$, dotted lines denote $\rho_{\chi}=\rho_{c}$, dot-dashed lines signify $\Gamma\left(Z \rightarrow \chi_{1}^{0} \chi_{1}^{0}\right)=\Gamma_{Z}^{i n v^{\prime}}$, and along the solid lines $\tilde{M}_{1}=\tilde{M}_{ \pm}$. Arrows point towards the region allowed by each individual constraint. 

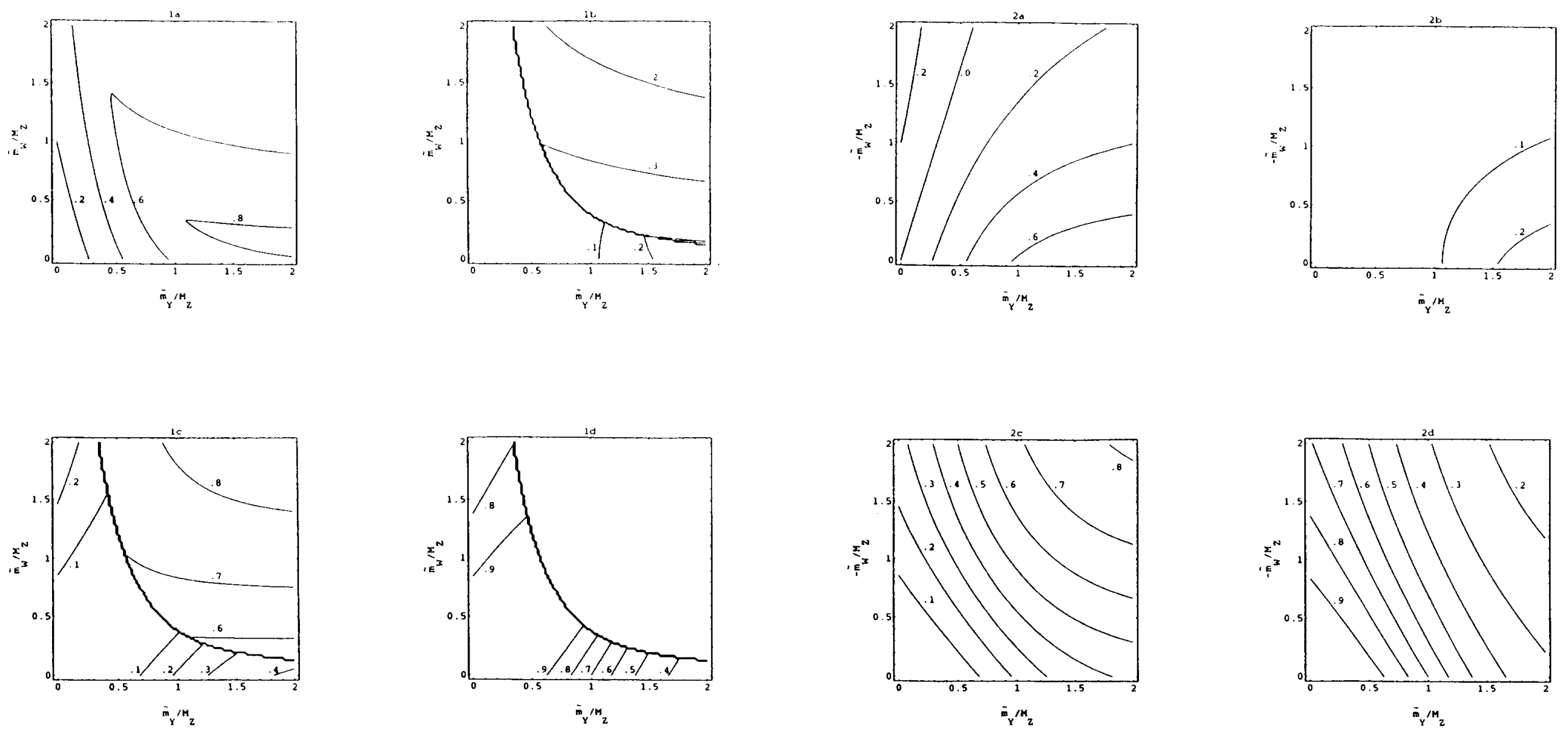

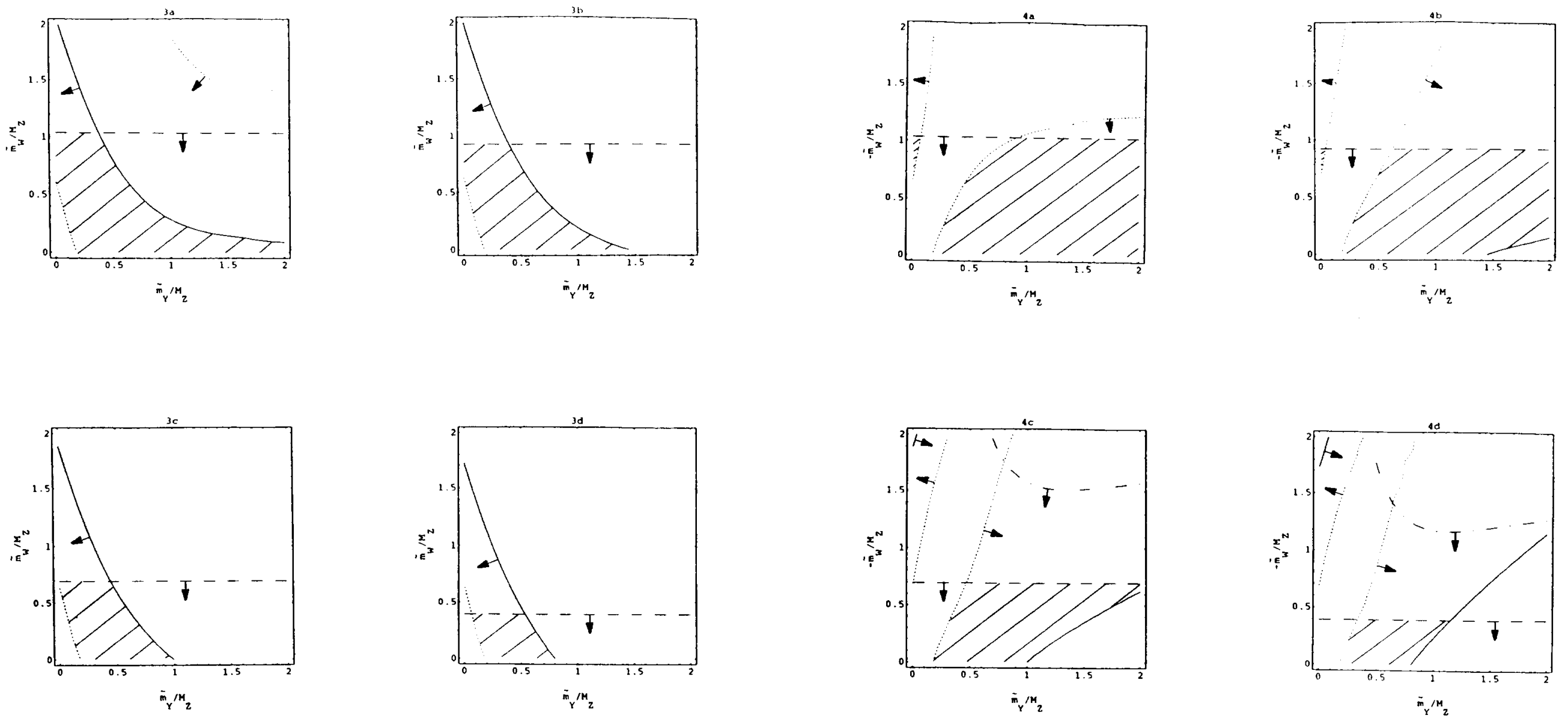